ORIOINAL BESEMRGI ARTIGLE

\title{
OUTCOME OF ACROMIO-CLAVICUALR JOINT DISLOCATION OPERATED USING CLAVICLE HOOK PLATE
}

Akesh Prajapati, Jyoti Sitaula, Bishnu Dev Sharma, Sujit Shrestha, Pujan Pant, Sanjeev Rijal ${ }^{1}$ Department of Orthopedics, Chitwan Medical College, Bharatpur, Nepal.

${ }^{*}$ Correspondence to: Dr. Akesh Prajapati, Department of Orthopedics, Chitwan Medical College, Bharatpur, Nepal. Email: akeshprajapati@hotmail.com

\begin{abstract}
Rockwood classification of AC joint dislocation is generally accepted worldwide. Treatment of Rockwood Type 1 and 2 AC joint is non-operative while all authors advocate operative treatment for Rockwood Type 4 and 5 AC joint dislocations. Thirty consecutive patients who underwent operation for acromioclavicular joint dislocation using clavicle hook plate from June 2015 were studied. The dislocations ranged from Rockwood type III to type V. Average follow up time was fourteen months ranging from four to twenty-four months. The outcome was measured using University of California Los Angeles (UCLA) shoulder score. All-the patient had good to excellent result.
\end{abstract}

Key words: Acromioclavicular joint, Clavicle hook plate

\section{INTRODUCTION}

Rockwood classification of AC joint dislocation is generally accepted worldwide. No controversy exists for treatment of Rockwood Type 1 and 2 AC joint, which is non-operative. Similarly, all authors advocate operative treatment for Rockwood Type 4 and 5 AC joint dislocations. However, numerous operative procedures have been reported in the literature, with varied results. Rockwood type 3 AC joint dislocations have generated the most of the controversies, with advocates of operative Vs nonoperative treatment. . $^{1,2,3,4}$

\section{METHODS}

In this retrospective study, thirty consecutive cases of $A C$ joint dislocation treated in our institution in a period of two years by $A C$ hook plate between June 2015 and July 2017 were studies. All patients were operated in beach chair position under general or regional (interscalene brachial plexus block) anesthesia. A curvilinear incision was made on top of the joint. The joint was exposed and debrided off of articular cartilage. A 3-hole hook plate is then implanted, with the hook first being placed under and posterior to the acromion process and the plate was placed on the lateral end of clavicle pressed inferiorly, thus reducing the joint. The plate was secured with 3 screws.

\section{POST-OPERATIVE MANAGEMENT}

Patients were placed on arm sling pouch for comfort. Early ROM exercise of the shoulder was started after forty-eight hours post- operatively. Sutures were removed two weeks post-operatively.

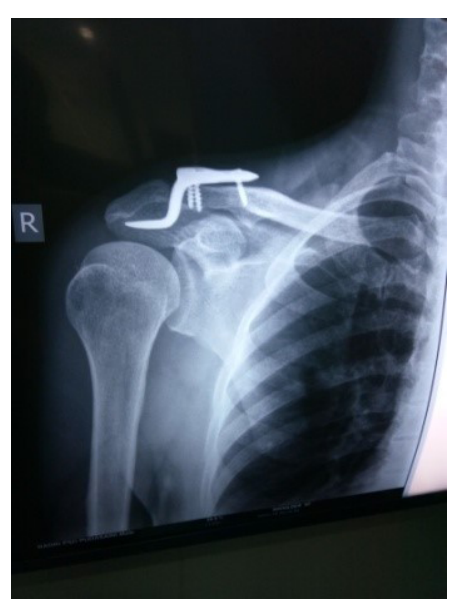

Fig 1: Pie Chart showing Sex distribution 


\section{RESULTS}

There were thirty patients, twenty males and ten females. Mean age was 49 years (25- 70 years). Sixteen right and fourteen left AC joint were injured. Mode of injury in all but one case was road traffic accident. In the remaining one patient mode of injury was fall from height. There were twenty four types 3 , four type 5 , one type 4 , and one AC dislocation with medial end clavicle fracture.

Average follow up was 14 months (4 - 24 months). All patients were rated according to UCLA Shoulder score. Twenty nine patients had good to excellent result. One patient had satisfactory result. Till now, no patient has complained of impingement, nor has any implant failure been noticed.

Two patients have under gone implant removal, after a period of one year post surgery. AC joint reduction was maintained after implant removal.

In this series all patient returned to work on average of 3 months (2-4 months).

\section{DISCUSSION \& CONCLUSION:}

AC joint dislocation is not uncommon. Though Rockwood classification is widely accepted, treatment plan (operative Vs non-operative) and various modalities of treatment has raised controversies in the literature. ${ }^{5}$

Over the past 30 years, many authors have supported non-operative treatment for complete $A C$ joint dislocations. ${ }^{6}$ Systemic review by Spencer concluded that non-operative treatment was superior to traditional operative treatment. ${ }^{7}$ Moreover, operative treatment was associated with higher complication rates, longer convalescence, and longer time away from sports and work. However, patients treated non-operatively for AC joint dislocation have unsatisfactory results, with residual pain during shoulder motion, loss of strength and cosmetic concerns.

To highlight the controversies further, following studies have supported operative treatment to have favorable outcome using different techniques.
Roper reported 15 cases of ORIF with $100 \%$ excellent results. ${ }^{8}$ Larsen reported of Phemister procedure in 39 patients with $97 \%$ good results. Our study also has similar results. $96 \%$ of the patient had excellent result. ${ }^{9}$

The aim of any operative procedure is to restore normal function and early return to work or sports. In our series, all the patients were able to return to their profession with normal or near normal range of motion of the shoulder joint.

\section{ACKNOWLEDGEMENT}

While the debate on management of $A C$ joint dislocation continues, we would like to conclude that satisfactory results can be obtained for operative management of $A C$ joint dislocation (type 3-5) using $A C$ hook plates.

\section{REFERENCES}

1. Darabos $\mathrm{N}$, Vlahovic $\mathrm{I}$, et al. Is AC tight rope fixationbetter than Bosworth screw fixation for minimally invasive operative treatment of Rockwood III AC joint injury? Injury Nov 2015;46(S):113-8.

2. An Wj, Sun Jb, et al. Comparative study of AC joint dislocation: Coracoclavicular ligament reconstruction combined with hook plate fixation or suture anchor fixation. Zhonghua wai Ke Za Zhi. 2013;51(4):349-53.

3. Calvo E, Lopez Franco M. Clinical and radiological outcome of surgical and conservative treatment of type III AC joint injury. Journal of Shoulder and Elbow surgery. 2006;15(3):300-305.

4. Galpin RD, Hawkins RJ. A comperative analysis of operative versus nonoperative treatment of grade III AC seperations. Clinical Orthopedics and Related Research. 1985;193:150-155.

5. Nissen CW, Chattejee A. Type III AC separation: result of a recent survey on its management. The American Journal of Orthopedics. 2007;38(2):89-93.

6. Press J, Zukerman JD. Treatment of grade III AC seperations: operative Vs nonoperative management. Bulletin: Hospital for joint diseases. 1997;56(2):7783. 
7. Spencer EE. Treatment of grade III AC joint injuries: a systemic review. Clinical Orthopedics and Related Research. 2007;45(5):38-44.

8. Roper BA, Levack B. The surgical treatment of acromioclavicular dislocations. The Journal of Bone and Joint Surgery. 1982;64(5):597-599.

9. B. Kiensat, R. Thietje, et al. Mid-term results after operative treatment of Rockwood Grade III- V AC joint dislocations with an AC Hook Plate. Eur J Med Res. 2011;16:52-56. 